\title{
Scanning Electron Microscopic Evaluation of Spray Coated Micro- Fibrillated Cellulose on the Packaging Paper
}

\author{
Kirubanandan Shanmugam \\ Department of Chemical Engineering, Bioresource Processing Institute of Australia, \\ Monash University, Clayton, Victoria -3800, Australia.
}

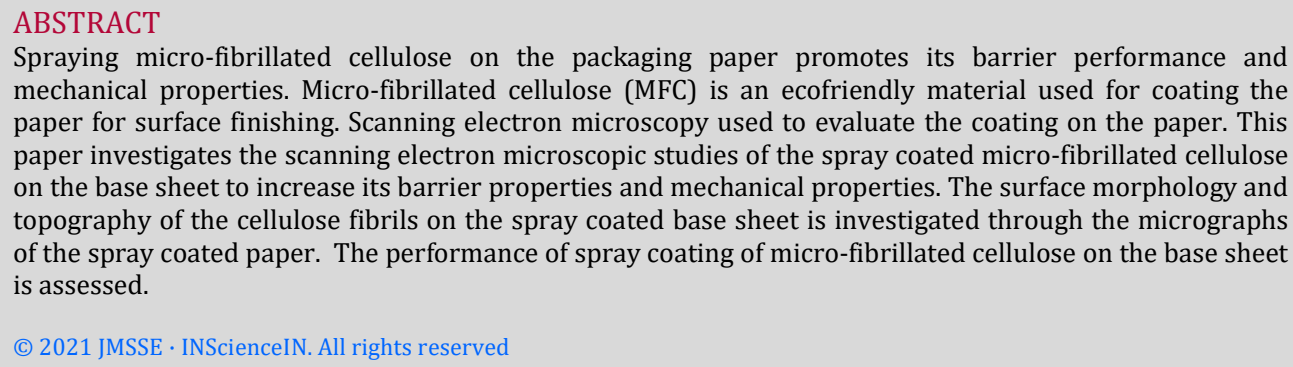
mechanical properties. Micro-fibrillated cellulose (MFC) is an ecofriendly material used for coating the paper for surface finishing. Scanning electron microscopy used to evaluate the coating on the paper. This paper investigates the scanning electron microscopic studies of the spray coated micro-fibrillated cellulose on the base sheet to increase its barrier properties and mechanical properties. The surface morphology and topography of the cellulose fibrils on the spray coated base sheet is investigated through the micrographs of the spray coated paper. The performance of spray coating of micro-fibrillated cellulose on the base sheet is assessed.

(C) $2021 \mathrm{JMSSE} \cdot$ INScienceIN. All rights reserved

\author{
ARTICLE HISTORY \\ Received 01-02-2021 \\ Revised 22-02-2021 \\ Accepted 23-02-2021 \\ Published 06-04-2021 \\ KEYWORDS \\ Micro fibrillated \\ Cellulose, \\ Spray coating, \\ Barrier coating and \\ Packaging paper
}

\section{Introduction}

The scanning electron microscopy is the most important tool for morphological and topographical analysis of the material surface at a suitable magnification. In this research, it is used to evaluate the surface morphology and topography of the spray coated micro-fibrillated cellulose on the base sheet through the investigation of different size of fibres and particles of cellulose on the base sheet. The digital images acquired in SEM, by the Secondary Electron Mode -1 and Mode II have suitable quality and resolution for further image processing and analysis to investigate the surface pores of the coated paper and size of the cellulose fibrils in the coated surface on the base sheet. The present investigation exemplifies how scanning electron microscopy does useful in assessing the performance of spray coating of micro-fibrillated cellulose and coating coverage on the base sheet and a coating layer/film on the base sheet.

Paper is widely used in packaging applications and is biodegradable and therefore perfectly safe for the environment. The hydrophilic nature of cellulose limits the water vapour-barrier properties and oxygen barrier properties of paper. To mitigate these limitations, paper is often associated with other materials, such as plastics, wax and aluminum, for their good barrier properties. However, these materials suffer from serious environmental issues, as difficult and inefficient to recycle. Recently, nanocellulose based materials has been considered as an alternative to produce eco-friendly barrier materials. Existing techniques to prepare nanocellulose films/sheets/composites are commercially not feasible and expensive. Therefore, other cost effective and readily implementable methodologies are required to achieve nanocellulose barrier layers. In the present thesis a novel approach is developed using spray coating technique to produce materials with excellent barrier properties [1]. Past decade, Coating is an alternative and novel process to increase the barrier properties and mechanical properties of the paper. Recent studies confirmed that the coating of micro-fibrillated cellulose on the base sheet enhance its barrier and mechanical properties of the base sheet [2-3]. Among many coating techniques, the spray coating has many advantages such as the production of even coating surface on the base sheet and contactless coating with the substrate.

Micro fibrillated cellulose is a bio-renewable and biodegradable material and the dimension of the diameter of 5-60 nm and several micrometres in length [4]. The spray deposition of nano-cellulose on the base sheet is assessed by coating coverage on the surface of the base sheet. The coating material coverage on the base sheet is evaluated by scanning electron microscopy investigation of the surface of the coated and uncoated base sheet [1]. A laboratory scale spray coating of nanocellulose suspension on a paper substrate was developed. When the nanocellulose suspension concentration was varied from 0.5 to $1.5 \mathrm{wt}$. \%, coat weight is increased from $2.9 \pm 0.7$ to $29.3 \pm 6.9 \mathrm{~g} / \mathrm{m}^{2}$. As a result, the air permeability of composite was decreased $0.78 \pm 0.17$ to $<0.0030 \mu \mathrm{m} /$ Pa.s. Scanning electron microscopy studies of spray coated paper confirms that the surface pores in the paper substrates are filled with sprayed nanocellulose and forms a continuous film on the surface of the substrate. These are the probable reasons for the reduction of air permeability of composites [1].

The micrographs of the surface of the spray coated paper are collected in the Secondary electron mode (Low-Mode I and High resolution -Mode -II) in FEI Nova SEM. The micrographs are used to study the micro/nanofibers orientation, cellulose particles and surface porosity of the coated paper. This paper investigates the scanning electron microscopic of the micro-fibrillated spray coated paper. Moreover, the image analysis of the micrographs using Image J software is performed. Using the image analysis of the micrographs, the fibre diameter is evaluated. 


\section{Experimental \\ Materials}

MFC (Micro-fibrillated cellulose) suspension as a coating formulation used for spray deposition on the base sheet [5]. Among many coating techniques, the spray coating is a novel technique and has many advantages such as the production of even coating surface in the base sheet and contactless coating with the substrate. This work investigates a lab scale spray coating of Micro fibrillated cellulose (MFC) which is a bio-based nano-material and diameter of fibre from 5-60 $\mathrm{nm}$ and length of several micrometres [4] on paper substrates and evaluated its barrier properties and mechanical properties. A laboratory scale spray coating of MFC suspension used from 0.25 to 1.5 wt. \% on the paper substrates is developed and assessed the coating performance on the base sheet by Scanning Electron Microscopy.

\section{Scanning Electron Microscopy of the coated papers: \\ Sample Preparation}

The spray coated paper ( $4 \mathrm{~mm} \mathrm{X} 4 \mathrm{~mm}$ ) is fixed on the stab using carbon tape and blowed with Nitrogen to remove the any dust or any loose material on the sample and then coated with Iridium with a maximum thickness of $10 \mu \mathrm{m}$. Moreover, the iridium coated samples are blowed off with Nitrogen to remove any dust and loose materials on the sample before loading into the FEI-NOVA Nano SEM 450 [6].

\section{Parameters for Scanning Electron Microscopy}

Micro fibrillated Cellulose is a biodegradable and delicate material in nature and highly susceptible to high accelerating voltage. Therefore, the parameters for collecting micrograph is optimized. The surface morphology and topography of the spray coated paper was characterized using FEI-NOVA Nano SEM 450 [6].

Mode 1: This mode is used for collecting the low resolution micrograph at $100 \mu \mathrm{m}$ and this micrograph is ideal for investigating the survey of the surface of the nanocellulose coated sheet and the roughness of the coated surface. The optimized parameters for high voltage and spot size are 3 $\mathrm{KV}$ and 2.00 respectively. The working distance and aperture size are $5 \mathrm{~mm}$ and $6(30 \mathrm{~mm})$.

Mode 2: This mode is used for collecting the micrograph at $1 \mu \mathrm{m}$ and $10 \mu \mathrm{m}$ (high resolution (UHR) imaging) and this micrograph is ideal for investigating the fibre orientation and size of the fibres and pores in the surface of the spray coated surface. The optimized parameters for high voltage and spot size are $3 \mathrm{KV}$ and 2.00 respectively. The working distance and aperture size are $5 \mathrm{~mm}$ and 6 (30 $\mathrm{mm}$ ) [7-9].

\section{Results and Discussion}

Spraying of micro fibrillated cellulose is an alternative technique to produce composite laminates by spraying onto a base sheet. Spraying has some significant advantages such as contour coating and contactless coating with the base substrate. In spray coating, the topography of the surface of the base substrate does not influence on the coating process. In addition to that, this coating has various advantages such as contactless, contour coating, coating of tear sensitive material and controlling the coating material. It is a novel technique for creating barrier film on the base surface rapid manner. Beneventiet. al. [5] reported that the laboratory scale spray coating of micro fibrillated cellulose on different kinds of paper substrate enhances the barrier and mechanical properties of the spray coated sheet. However, after spraying, they used vacuum filtration which is like the conventional paper making process to remove the excess water. Therefore, it leads to time consuming process.

The spraying of Micro fibrillated cellulose as laminates on the packaging paper in a continuous mode is not explored so far. The micro fibrillated cellulose as laminated on the base sheet enhance the barrier properties of the sheet for packaging applications. Previous study reported the batch process of spray coating of micro fibrillated cellulose on the paper, in this process, after spray coating, vacuum filtration is used to remove the excess water in the sprayed nanocellulose on the base sheet [5]. The simplified continuous spray coating nanocellulose on the base sheet is not explored so far. Figure 1 shows the developed spray coating process on the coating of MFC on the packaging paper.

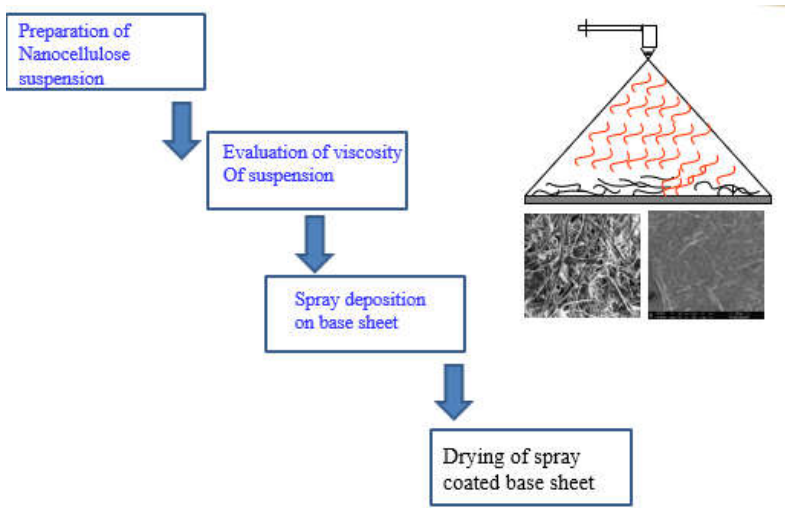

Figure 1: Steps in spraying MFC on the packaging paper

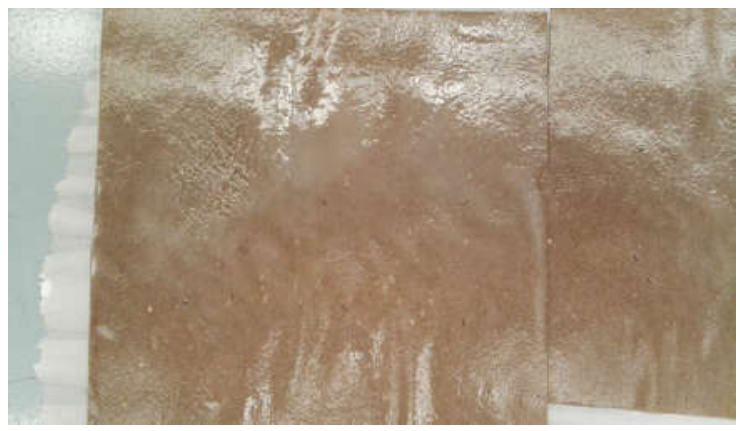

Figure 2: Sprayed MFC on the packaging paper

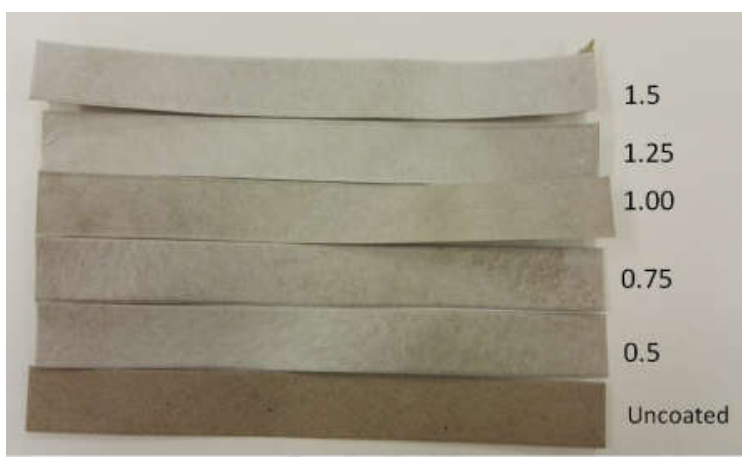

Figure 3: MFC deposited on the packaing paper with different concentration 


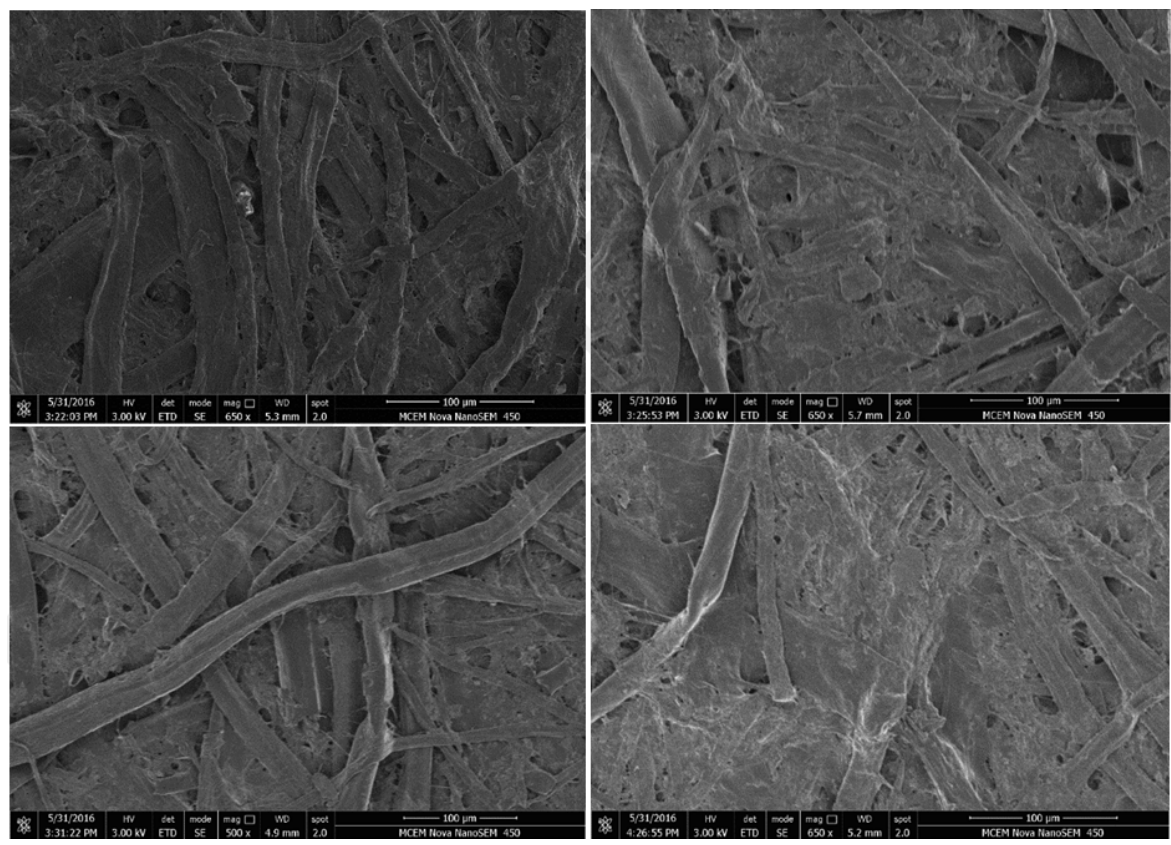

Figure 4: The plain brown paper magnification at $100 \mu \mathrm{m}$

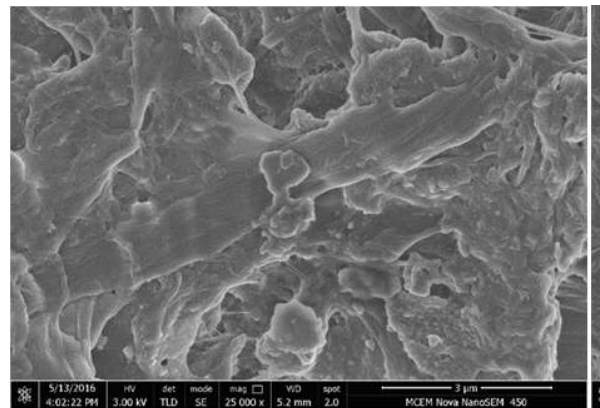

Figure 5.A. 0.25 Wt. \% MFC Coated

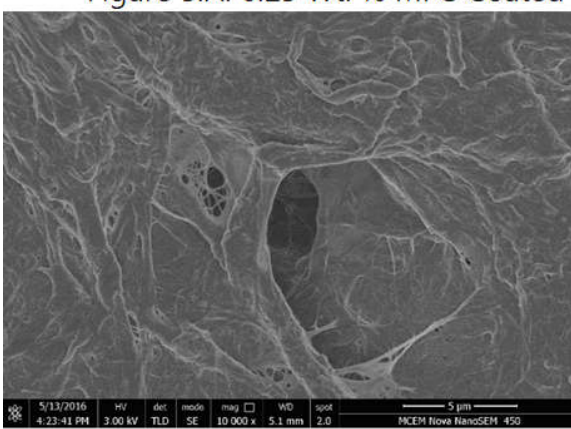

Figure 5.C. 0.75 Wt. \% MFC Coated

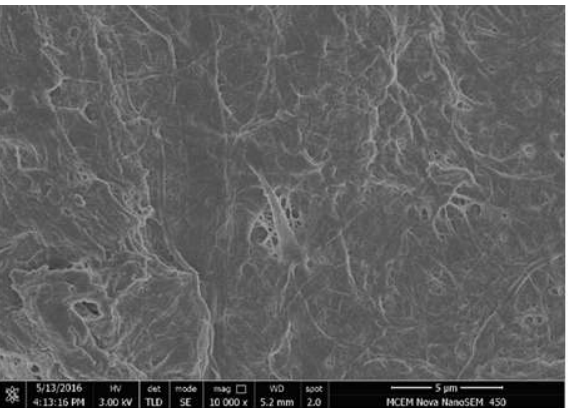

Figure 5.B. 0.5 Wt.\% MFC Coated

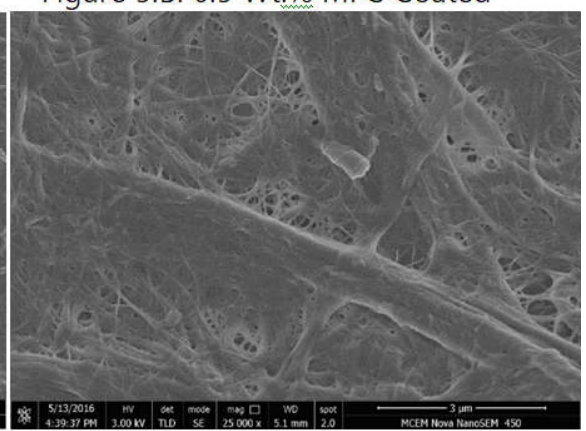

Figure 5D. 1.00 Wt. \%MFC Coated

Figure 5: MFC coating on the packaging paper

Figure 2 shows the wet lamination of MFC on the packaging paper via spray coating. It shows the contour coating of micro-fibrillated cellulose on the packaging paper. Figure 3 shows the dried sheet after spray coating of Micro fibrillated cellulose. Visually Fig. 3 confirmed even lamination of MFC on the packaging paper via spraying process. Sprayed micro-fibrillated cellulose on the packaging paper filled surface pores in the packaging paper. As a result, the barrier performance of the spray coated packaging paper was boosted and air permeance of the sheet decreased with increased coating concentration of MFC on the sheet. Scanning electron microscopy is played as a quality tool for coating of MFC on the packaging sheet. This method confirms the lamination performance of MFC on the packaging paper. Figure 4 shows the secondary electron image at low magnification of the uncoated paper and shows the network structure of cellulose fibres on the surface of the paper at low magnification. The micrograph of uncoated paper shows the presence of various size of surface pores. Figure 4 A shows clearly visible of network structure of the cellulose fibrils and surface pores in the uncoated paper. Figure 4 B shows the fillers occupied the space between two fibres in the surface of the paper. Figure $4 \mathrm{C}$ shows the complete network structure of the cellulose fibres in the surface of the paper. Figure $4 \mathrm{D}$ confirms the fillers occupies the space between fibres in the paper. The cellulose fibres in the uncoated paper is $13.68 \pm 3.27 \mu \mathrm{m}$. The mean size of the pores in the surface of the uncoated sheet is $8.36 \pm 1.44 \mu \mathrm{m}$. 

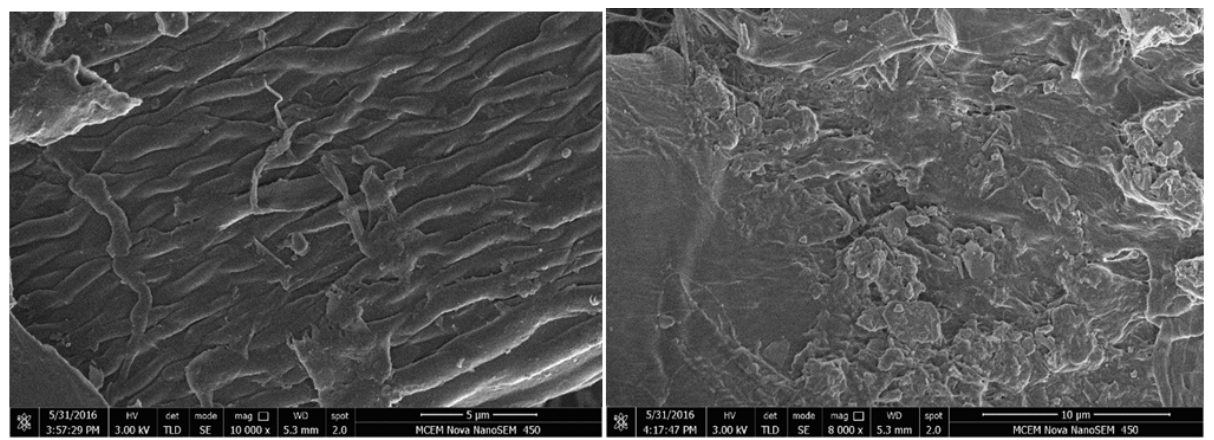

Figure 6: Micrographs of the plain paper (uncoated paper) at $5 \mu \mathrm{m}$ and $10 \mu \mathrm{m}$. The brown paper (uncoated) contains fillers usually calcium carbonate and agglomerates of fillers observed as a feature in the surface of the paper at high magnification
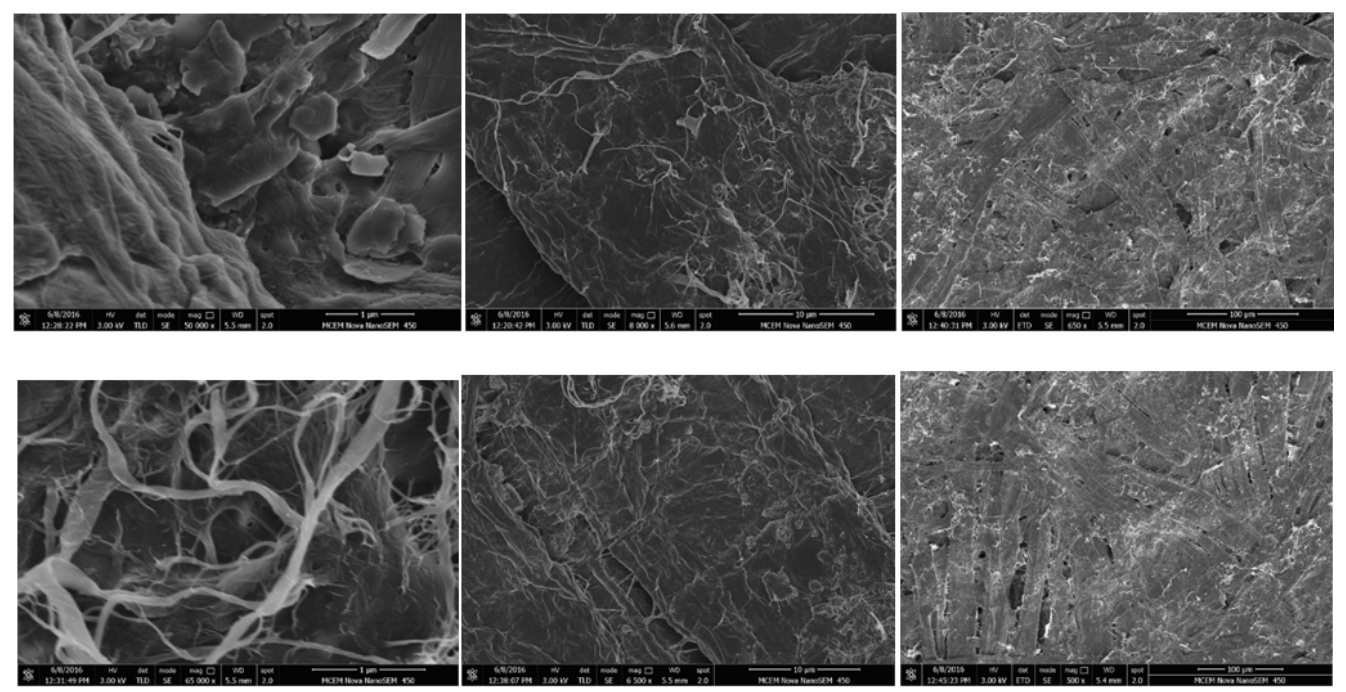

Figure 7: Micrographs of the spray coated paper with 1.25 wt. \% of the micro-fibrillated cellulose
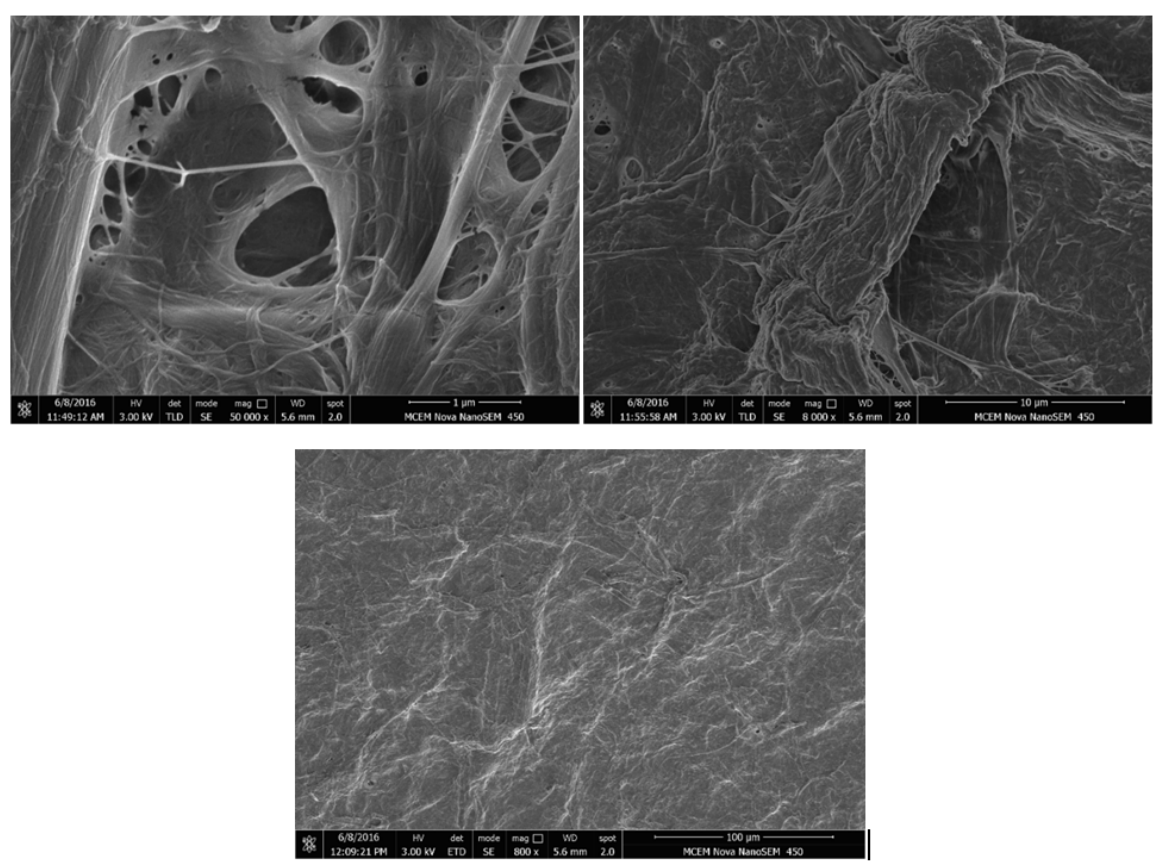

Figure 8: Micrographs of the micro fibrillated cellulose film peeled from the spray coated paper

The Figure 5 shows the surface morphology of the paper spray coated with different concentration of Microfibrillated cellulose on the base sheet. The Figure 5 A shows the surface of the spray coated paper with $0.25 \mathrm{Wt} \%$ of Micro-fibrillated cellulose. It shows the large clumps of the cellulose fibres on the base sheet. Micro-fibrillated cellulose contains particle and different size of the fibres. Figure 5 B shows the spray coated with $0.5 \mathrm{Wt}$. \% paper and the deposited micro fibrillated cellulose on the base sheet forms homogenous film with different size fibres and 
particles. The cellulose film on the spray coated paper has many surface pores. Figure $5 \mathrm{C}$ shows the spray coated with $0.75 \mathrm{Wt}$. \% paper and the homogeneous films are formed by the deposited MFC on the base sheet, the films contains different size of fibres and particles with surface pores. Figure $5 \mathrm{D}$ shows the spray coated with $1.00 \mathrm{Wt}$. \% paper and the homogeneous films with a cellulose fibrils network on the spray coated paper is formed.

In figure $5 \mathrm{~A}$, the size of the micro fibres in the $0.25 \mathrm{wt} \%$ coated at high magnification varies from $1.23 \mu \mathrm{m}$ to 0.094 $\mu \mathrm{m}$ in the diameter. The average size of the surface pores is $0.17 \mu \mathrm{m}$. In figure $5 \mathrm{~B}$, the average diameter of the micro fibre is $0.21 \mu \mathrm{m}$. The size of the surface pores ranged from $1.149 \mu \mathrm{m}$ to $0.34 \mu \mathrm{m}$.

The Fig. 6 shows the micrograph of uncoated paper collected at high magnification in SE Mode II. The micrograph confirms the complete network of cellulose fibrils in the surface of the paper. The paper surface contains fillers such as Calcium Carbonate to increase its opacity [10]. Figure $6 \mathrm{~B}$ shows the particles of fillers on the paper surface. These fillers interacted with fibre void space.

The Figure 7 shows the micrograph of the spray coated paper with $1.25 \mathrm{Wt}$. \% of the micro-fibrillated cellulose at high and low magnification. The micrograph $(1 \mu \mathrm{m})$ shows the deposited clumps of cellulose fibres and cellulose fibres on the base sheet and confirmed the presence of different size of the fibre entangled with cellulose fibres clumps. The micrograph $(10 \mu \mathrm{m})$ shows the different size of the cellulose fibres and formed layer on the base sheet. Moreover, the micrograph $(100 \mu \mathrm{m})$ confirms the complete coverage of micro-fibrillated cellulose coating formulation on the base sheet. When compared to the micrograph $(100$ $\mu \mathrm{m}$ ) of the uncoated paper, the coated paper showed that the coating formulation filed many surface pores and void space between the cellulose fibres.

The Figure 8 shows the micrograph of the micro-fibrillated cellulose film peeled from the spray coated paper. The cellulose film has pores in the surface and consists of the different size of the cellulose fibres in the film. The cellulose film has surface roughness due to the presence of different size of cellulose fibres. Figure $8 \mathrm{~A}$ : It confirms the presence of the various size of pores in the surface of the film. It is observed that there are many discontinuity in the cellulose fibres. The different size of the fibres and pores are distributed randomly in the cellulose film. It confirms the random fibres network at microscale. The size of the pores in the films are irregular shape and the cellulose fibres are stretched on the surface of the film. The clear networks of the surface pores are observed. Figure 8B: It shows clumps of different size of the fibres in the film. While spray deposition of nano cellulose on the base sheet, the deposited cellulose fibrils form aggregates due to the inter-fibrillary hydrogen bonding. Figure 8C: Thefilm has roughness in the surface due to the presence of the different size of the fibres.The surface roughness of the cellulose films seems to be caused by residual cellulose micro and nanofibers and it creates the uneven surface in the film

\section{Conclusions}

The SEI micrographs of the spray coated paper confirms that the spray deposition of micro fibrillated cellulose on the base sheet provides good coating coverage of the base sheet surface and filled the surface pores on the surface of the base sheet. The spray coating of MFC does seem to form a more closed structure homogenous cellulose nanofiber film on the base sheet compared with the uncoated sheet.

\section{Significance}

The formation of nanocellulose film via spraying on the packaging paper promotes its barrier performance and mechanical properties. The spray coated nanocellulose on the base sheet fills the surface pores on the sheet and promotes its barrier performance. SEM micrographs can be used to test the quality of spray coated nanocellulose film on the sheet such as uniformity, morphology of spray coated nanofibers on the sheet and texture on the surface.

\section{Acknowledgement}

I would like to acknowledge the facilities used with the Monash Centre for Electron Microscopy. K. S. is grateful to Monash University, Bioprocessing Research Institute of Australia and Bioprocessing Advanced Manufacturing Initiative and Faculty of Engineering International Postgraduate Research Scholarship. I also acknowledge Associate Professor Warren Batchelor and Professor Gil Garnier for the supervision of my doctoral research in spray coating.

\section{References}

1. S. Kirubanandan, Development of Microfibrillated cellulose based functional material via spray coating - Seminar Report, Submitted to Department of Chemical Engineering, Monash University, Victoria, Australia, 2016.

2. S. Kirubanandan, Development of a spray coating process to enhance the barrier properties of a base sheet Presented in BAMI Industry Transformation Research Hub, Australian Pulp and Paper Institute, Department of Chemical Engineering, Monash University, Australia, 2016.

3. S. Kirubanandan., Enhancing the barrier properties of a base sheet using a spray coating of micro -fibrillated cellulose Presented in BAMI Student Chapter, Australian Pulp and Paper Institute, Department of Chemical Engineering, Monash University, Australia, 2016.

4. D. Klemm et al., Microfibrillated celluloses: A New Family of Nature-Based Materials. Angewandte Chemie International Edition, 2011,50(24), 5438-5466.

5. D. Beneventi et al., Highly Porous Paper Loading with Microfibrillated Cellulose by Spray Coating on Wet Substrates. 2014, Industrial \& Engineering Chemistry Research 53 (27), 10982-10989.

6. J. Ma., SEM Sample Preparation. Monash Centre for Electron Microscopy, Course Notes, Monash University, Australia, 2015.

7. F. Burgmann., Introduction to Scanning Electron Microscopy: Theory and Applications in Physical Sciences". Monash Centre for Electron Microscopy, Monash University, Australia, 2016.

8. A Technical Report on "Scanning Electron Microscope Training Module", Australian Microscopy \& Microanalysis Research Facility.

9. Manager, Nova., User's Quick Reference Guide Version 3.0 for FEI Nova NanoSEM 450 Field Emission Scanning Electron Microscope, Monash centre for Electron Microscopy, Monash University, Clayton, Australia, 2015.

10. A. Mikko et al., The Physics of Paper, Report on Progress in Physics, 2006, 69, 669-723. 